\title{
Rho-Related GTP-Binding Protein RhoC
}

National Cancer Institute

\section{Source}

National Cancer Institute. Rho-Related GTP-Binding Protein RhoC. NCI Thesaurus. Code C17416.

Rho-related GT P-binding protein RhoC (193 aa, $22 \mathrm{kDa}$ ) is encoded by the human RHOC gene. This protein is involved in the assembly of focal adhesions and stress fibers. 